\title{
NOTES
}

\section{Butyrate Esterase (Tributyrin) Spot Test, a Simple Method for Immediate Identification of Moraxella (Branhamella) catarrhalis}

\author{
JOSÉ L. PÉREZ,* ANGELES PULIDO, FLORENCIA PANTOZZI, AND ROGELIO MARTIN \\ Service of Microbiology, Hospital de Bellvitge Principes de España, Universidad de Barcelona, \\ 08907 Hospitalet de Llobregat, Barcelona, Spain
}

Received 28 March 1990/Accepted 28 June 1990

\begin{abstract}
A total of 47 Moraxella (Branhamella) catarrhalis strains, 89 strains of Neisseria spp., and 82 strains of miscellaneous bacteria and yeasts were studied by using a fluorogenic spot method which detects butyrate esterase. A positive butyrate esterase spot test correctly differentiated $M$. catarrhalis from Neisseria spp., which had a negative butyrate esterase spot test reaction. The test is rapid, simple, and easy to perform. The butyrate esterase spot test was useful for direct identification of $M$. catarrhalis from primary cultures. However, false-positive reactions may occur with mixed cultures.
\end{abstract}

Moraxella (Branhamella) catarrhalis is now recognized as a major pathogen that causes otitis media, sinusitis, conjunctivitis, bronchitis, and pneumonia. In addition, a significant proportion of $M$. catarrhalis strains produce the enzyme $\beta$-lactamase. This organism shares several characteristics with the saprophytic strains of Neisseria spp. Both are found in the same habitat, and both are asaccharolytic. Conventional tests may require 24 to $48 \mathrm{~h}$ for completion. Alternative rapid, easy-to-perform identification methods are desirable.

The differences in the esterase activities of members of the family Neisseriaceae have been known for several years. From the early studies of Berger (1), tributyrin (glycerol tributyrate) hydrolysis has been used as a key test for differentiation and identification of $M$. catarrhalis $(4,8)$. Several substrates and techniques have been proposed for detecting butyrate esterase $(2,3,6)$. Vaneechoutte et al. (7) recently described a fluorogenic method that allowed accurate results after $5 \mathrm{~min}$ of incubation. The aim of this study was to evaluate the usefulness of a spot fluorescence technique which simplifies and shortens this method.

A total of $47 \mathrm{M}$. catarrhalis strains, 89 strains of Neisseria spp., and 82 strains of other bacteria and yeasts were studied (Table 1). All of the strains were isolated from human sources at different hospitals, except for 26 strains that were kindly supplied by the reference laboratory Centro Nacional de Microbiología, Majadahonda, Madrid, Spain. Most of the strains were maintained frozen at $-70^{\circ} \mathrm{C}$ or lyophilized. Stock strains were subcultured twice to appropriate media before testing.

Neisseria spp. and $M$. catarrhalis strains were identified by using standard methods $(5,8)$. The tests which we used included Gram staining and tests for oxidase, catalase, superoxol (4), DNase, nitrate reduction, susceptibility to a $10-\mu \mathrm{g}$ colistin disk, and production of acid from glucose, maltose, lactose, sucrose, and fructose. $\gamma$-Glutamyl aminopeptidase and $o$-nitrophenyl- $\beta$-D-galactopyranoside detection tests were performed when they were needed. The

\footnotetext{
* Corresponding author
}

remaining bacterial strains and yeasts were identified by using conventional techniques. Butyrate esterase activity was detected by using the following methods: the acidimetric method, the tube fluorogenic method, and the butyrate esterase spot test (BST).

In the acidimetric method (substrate, tributyrin), tests were performed by using the technique of Riou et al. (6) and commercially available tributyrin tablets (Rosco, Taastrup, Denmark). A heavy suspension of an organism that was equivalent to a no. $2 \mathrm{McF}$ arland standard was made in a screw-cap tube containing $0.5 \mathrm{ml}$ of saline. One tributyrin tablet was added to the suspension, and the preparation was mixed and incubated at $35^{\circ} \mathrm{C}$. Tests were read after 2,3 , and $4 \mathrm{~h}$ of incubation (Table 1). The development of bright yellow was considered to be a positive result. Each batch of tablets was quality controlled with the following reference strains: $M$. catarrhalis ATCC 25238 (positive) and Neisseria meningitidis ATCC 13090 (negative).

In the tube fluorogenic method (substrate, methylumbelliferyl butyrate), tests were performed as described by Vaneechoutte et al. (7). Briefly, the reagent was prepared as follows: a stock solution was made by dissolving $100 \mathrm{mg}$ of 4-methylumbelliferyl butyrate (Sigma Chemical Co., St. Louis, Mo.) in $10 \mathrm{ml}$ of dimethyl sulfoxide (E. Merck AG, Federal Republic of Germany) and $100 \mu$ l of Triton X-100 (Sigma). The stock solution was diluted $1: 10$ in $0.1 \mathrm{M}$ McIlvaine citrate buffer (pH 5.0); this preparation could be stored in $500-\mu$ l portions in glass tubes at $-70^{\circ} \mathrm{C}$ for at least 1 month. Two to three colonies from a recent culture plate (not older than $24 \mathrm{~h}$ ) were suspended in $0.25 \mathrm{ml}$ of fluorogenic reagent and incubated at room temperature. A positive test was indicated by the development of blue fluorescence after exposure to a 366-nm UV source. Tests were read after $5 \mathrm{~min}, 15 \mathrm{~min}$, and $18 \mathrm{~h}$ of incubation. The reference strains mentioned above and a reagent blank were used as controls in each test.

In the BST (substrate, methylumbelliferyl butyrate), the reagent was the same as that used for the tube fluorogenic method. A few drops of the reagent were placed onto a piece of filter paper. Two to three colonies from a recent culture were streaked onto the paper, which was immediately ex- 
TABLE 1. Detection of butyrate esterase by three methods

\begin{tabular}{|c|c|c|c|c|c|c|c|c|c|}
\hline \multirow{3}{*}{$\begin{array}{c}\text { Taxon } \\
\text { (no. of strains tested) }\end{array}$} & \multicolumn{9}{|c|}{ Cumulative no. of positive results ${ }^{a}$} \\
\hline & \multicolumn{3}{|c|}{ Acidimetric } & \multicolumn{3}{|c|}{ Tube fluorogenic } & \multicolumn{3}{|c|}{ BST method } \\
\hline & $2 \mathrm{~h}$ & $3 \mathrm{~h}$ & $4 \mathrm{~h}$ & $5 \min$ & $15 \mathrm{~min}$ & $>18 \mathrm{~h}$ & $30 \mathrm{~s}$ & $1 \mathrm{~min}$ & $3 \mathrm{~min}$ \\
\hline Moraxella catarrhalis (47) & 46 & 47 & & 47 & & & 47 & & \\
\hline Neisseria gonorrhoeae (27) & & & & & & 27 & & & 8 \\
\hline Neisseria meningitidis (28) & & & & & & 28 & & & 7 \\
\hline Neisseria lactamica $(12)$ & & & & & & 12 & & & 3 \\
\hline Neisseria polysaccharea (5) & & & & & & 5 & & & 1 \\
\hline Neisseria subflava $(7)$ & & & & & & 7 & & & 3 \\
\hline Neisseria sicca $(5)$ & & & & & & 5 & & & \\
\hline Neisseria denitrificans (1) & & & & & & 1 & & & \\
\hline Neisseria cinerea & & & & & & 3 & & & 1 \\
\hline Neisseria mucosa (1) & & & & & & 1 & & & \\
\hline Moraxella spp. (7) & 2 & 2 & 3 & 3 & 3 & 5 & 3 & 3 & 5 \\
\hline Acinetobacter spp. (27) & 23 & 25 & 25 & 14 & 24 & 27 & & 25 & 25 \\
\hline Pseudomonas aeruginosa (10) & 6 & 7 & 9 & 10 & & & & 10 & \\
\hline Staphylococcus aureus (10) & 8 & 10 & & 10 & & & 10 & & \\
\hline Coagulase-negative staphylococci (10) & 4 & 10 & & 10 & & & 10 & & \\
\hline Candida albicans $(10)$ & 9 & 10 & & 10 & & & 10 & & \\
\hline
\end{tabular}

${ }^{a}$ Positive results within the times indicated. There were no positive results for eight Eikenella corrodens strains.

posed to a UV lamp. A blue fluorescent spot was considered positive. The tests were read after $30 \mathrm{~s}, 1 \mathrm{~min}$, and $3 \mathrm{~min}$. Positive and negative reference strains were used as controls.

Table 1 shows the results obtained with the three methods at the intervals of incubation used. As expected, the acidimetric and tube fluorogenic techniques clearly differentiated between Neisseria spp. and $M$. catarrhalis strains when the tests were read after $3 \mathrm{~h}$ and $5 \mathrm{~min}$, respectively. All $M$. catarrhalis strains gave positive results with the BST within $30 \mathrm{~s}$. In contrast, enzymatic activity was not detected in Neisseria spp. by the BST, except for a few strains that showed weak reactions after $3 \mathrm{~min}$, probably because of nonspecific hydrolysis of the substrate. Three of seven strains of Moraxella spp. other than $\boldsymbol{M}$. catarrhalis also gave positive results with each of the three test methods. We found complete agreement between the BST results and the results of the reference techniques.

Among the remaining miscellaneous bacterial and fungal isolates, butyrate esterase activity was observed in Acinetobacter spp., Pseudomonas aeruginosa, staphylococci, and Candida albicans. Both staphylococci and $C$. albicans gave more rapid positive results than gram-negative rod-shaped bacteria.

In order to evaluate the possible interference of other microorganisms when the BST was performed, two to three colonies of six different strains of Neisseria spp. (three $N$. meningitidis strains, one $N$. lactamica strain, and two $N$. subflava strains) were mixed with two or three colonies of either Staphylococcus aureus or C. albicans. The mixed cultures were tested by using the BST. Each combination of microorganisms was tested in triplicate. False-positive results were obtained with all combinations after mean times of $\leq 42 \mathrm{~s}$. In the majority of cases, a fluorescent spot was observed within $30 \mathrm{~s}$.

In this study we demonstrated the utility of the BST. This is a technique that is simple and easy to perform and that gives immediate and accurate results. Moreover, it is also inexpensive in reagents and handling times. At current prices and exchange rates in Spain, each test (including two controls) costs approximately $\$ 0.10$. The proposed test may be used with confidence to differentiate $M$. catarrhalis from
Neisseria spp. in pure cultures. The BST is also useful for identifying $\boldsymbol{M}$. catarrhalis from primary isolation plates. In this case, however, caution must be taken with mixed cultures. The tests should be performed only on colonies of oxidase-positive, gram-negative diplococci. The morphology of the colonies and Gram staining results may also be helpful for differentiating $M$. catarrhalis from other Moraxella spp. that give positive BST results. The possibility of falsepositive results with the BST must be kept in mind when staphylococci and $C$. albicans are present, which may be difficult to detect when the colonies are immature.

We thank Julio Vázquez and Juan A. Sáez-Nieto, Centro Nacional de Microbiología, Majadahonda, Madrid, Spain, as well as other Spanish microbiologists who kindly supplied several strains tested in this study.

\section{LITERATURE CITED}

1. Berger, U. 1962. Ueber die Spaltung von Tributyrin durch Neisseria. Arch. Hyg. Bakteriol. 146:388-391.

2. Dealler, S. F., M. Abbott, M. J. Croughan, and P. M. Hawkey. 1989. Identification of Branhamella catarrhalis in $2.5 \mathrm{~min}$ with an indoxyl butyrate strip test. J. Clin. Microbiol. 27:1390-1391.

3. Janda, W. M., and P. Ruther. 1989. B.CAT CONFIRM, a rapid test for confirmation of Branhamella catarrhalis. J. Clin. Microbiol. 27:1130-1131.

4. Knapp, J. S. 1988. Historical perspectives and identification of Neisseria and related species. Clin. Microbiol. Rev. 1:415-431.

5. Morello, J. A., W. M. Janda, and M. Bohnhoff. 1985. Neisseria and Branhamella, p. 176-192. In E. H. Lennette, A. Balows, W. J. Hausler, Jr., and H. J. Shadomy (ed.), Manual of clinical microbiology, 4th ed. American Society for Microbiology, Washington, D.C.

6. Riou, J. Y., J. Buissière, M. Guibourdenche, G. Brault, and J. P. Carlier. 1981. Hydrolyse de la tributyrine par les Neisseria et les Branhamella. Ann. Microbiol. (Inst. Pasteur) 132A:159-169.

7. Vaneechoutte, M., G. Verschraegen, G. Claeys, and P. Flamen. 1988. Rapid identification of Branhamella catarrhalis with 4methylumbelliferyl butyrate. J. Clin. Microbiol. 26:1227-1228.

8. Vedros, N. A. 1984. Genus I. Neisseria Trevisan 1885 , p. 290 296. In N. R. Krieg and J. G. Holt (ed.), Bergey's manual of systematic bacteriology, vol. 1. The Williams \& Wilkins Co., Baltimore. 